\title{
Colitis-associated colorectal adenocarcinomas are frequently associated with non-intestinal mucin profiles and loss of SATB2 expression
}

\author{
Mai Iwaya ${ }^{1,2} \cdot$ Hiroyoshi Ota $^{3} \cdot$ Yoko Tateishi ${ }^{1} \cdot$ Tomoyuki Nakajima $^{4} \cdot$ Robert Riddell $^{1,2} \cdot$ James R. Conner $^{1,2}$
}

Received: 21 September 2018 / Revised: 18 November 2018 / Accepted: 19 November 2018 / Published online: 1 February 2019

(c) United States \& Canadian Academy of Pathology 2019

\begin{abstract}
The special AT-rich sequence binding protein 2 (SATB2) is a sensitive and specific diagnostic marker for colorectal adenocarcinoma and reduced expression of SATB2 is associated with a poor prognosis. Colitis-associated colorectal adenocarcinoma often shows distinct morphologic and molecular phenotypes compared to sporadic cases. However, the SATB2 expression profile in colitis-associated carcinoma has not been defined. We performed immunohistochemistry for SATB2 as well as CDX2, MUC5AC, MUC6 and mismatch repair proteins on 60 consecutive colitis-associated carcinomas from 58 inflammatory bowel disease patients and compared the expression profile to a control group of 32 sporadic colorectal carcinomas. Only 26 (43\%) colitis-associated carcinomas expressed SATB2, compared to 29 (91\%) sporadic colorectal carcinomas $(p<0.0001)$. MUC5AC expression was more frequently observed in colitis-associated carcinomas than sporadic colorectal caracinomas $(52 \%$ and $25 \%$ respectively; $p=0.013)$. Eight $(13 \%)$ cases of colitis-associated carcinoma showed loss of CDX2 expression, which was retained in all of the sporadic controls $(p=0.047)$. In colitisassociated carcinoma, 50\% of SATB2 negative cases had lymph node metastasis compared to only $15 \%$ of SATB2 positive cases $(p=0.007)$. Loss of SATB2 was particularly frequent in mucinous-type tumors, occurring in $83 \%$ of these cases. There was no significant association between SATB2 expression and mismatch repair protein status. These data show that the immunoprofile of colitis-associated carcinoma is different than that seen in sporadic cases. In particular, SATB2 is significantly less sensitive in colitis-associated carcinoma and it should be interpreted cautiously as a marker of colorectal origin in colitis patients. The association between loss of SATB2 and lymph node metastasis suggests that it may have similar prognostic value in the setting of inflammatory bowel disease as in sporadic cases.
\end{abstract}

This work was presented in part at the $107^{\text {th }}$ annual meeting of the United States and Canadian Society of Pathology in Vancouver, Canada, March 17-23, 2018.

James R. Conner

James.Conner@sinaihealthsystem.ca

1 Department of Pathology and Laboratory Medicine, Sinai Health System, Toronto, Canada

2 Department of Laboratory Medicine and Pathobiology, University of Toronto, Toronto, Canada

3 Department of Clinical Laboratory Sciences, School of Health Sciences, Shinshu University, Matsumoto, Japan

4 Department of Laboratory Medicine, Shinshu University Hospital, Matsumoto, Japan

\section{Introduction}

Patients with inflammatory bowel disease have an increased risk of colorectal adenocarcinoma that is proportional to disease duration [1], extent [2], and severity [3]. One metaanalysis estimated that the risk of colitis-associated carcinoma in patients with inflammatory bowel disease is $2 \%$ by 10 years after initial diagnosis, and increases to $8 \%$ at 20 years and $18 \%$ at 30 years after colitis onset [1]. The molecular pathogenesis of colitis-associated carcinoma is different than that of sporadic (i.e., non-colitis, nonsyndromic associated) colorectal adenocarcinoma, with genomic changes that appear directly linked to the effects of ongoing inflammation and repeated mucosal injury in the setting of inflammatory bowel disease $[4,5]$.

Special AT-rich sequence-binding protein 2 (SATB2), a nuclear matrix-associated transcription factor and epigenetic regulator, was initially identified as a gene involved in 
Fig. 1 Colitis-associated adenocarcinomas were morphologically classified as follows: conventional type, showing typical features of sporadic colitis-associated carcinoma (a), mucinous type, showing $>50 \%$ mucin production (b), serrated type, showing eosinophilic glands with serrated architecture infiltrating into the stroma (c), and low grade tubuloglandular type, showing very well-differentiated glands infiltrating into the stroma (d)
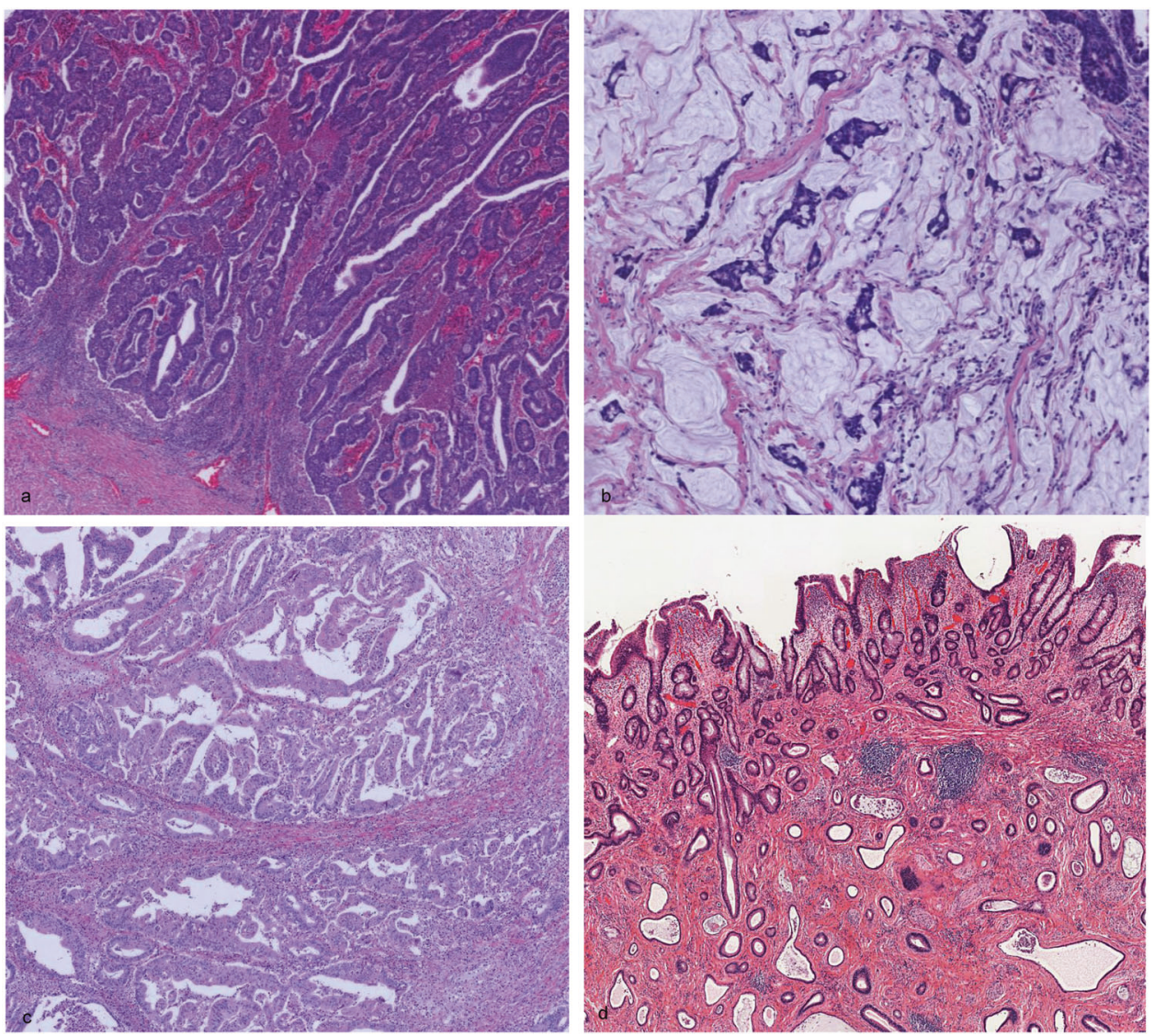

osteoblast differentiation and craniofacial patterning in humans $[6,7]$. SATB2 is normally expressed in the large intestinal epithelium and has diagnostic utility as a relatively specific immunohistochemical marker for colorectal adenocarcinoma [8-10]. Loss of SATB2 expression is associated with poor prognosis in colorectal adenocarcinoma $[11,12]$.

Given the unique genetic underpinnings of colitisassociated carcinoma, it follows that the immunoprofile of these tumors may not precisely mirror their sporadic counterparts, which could have important diagnostic (and potentially prognostic) consequences. Although prior studies have suggested that colitis-associated intestinal carcinomas tend to express the gastric-phenotype mucins MUC5AC and MUC6 at a higher rate than sporadic controls $[13,14]$, the larger of these series focused on small intestinal cancers and the number of colitisassociated carcinoma cases was relatively limited. To our knowledge, a large series comparing the immunoprofile of colitis-associated carcinoma to sporadic colorectal adenocarcinoma, and in particular the rate of SATB2 expression, has not been performed. Here, a series of consecutive colitis-associated carcinoma cases was compared with sporadic colorectal adenocarcinoma for histomorphologic features and the expression of SATB2,
CDX2, gastric phenotype mucins MUC5AC and MUC6, and mismatch repair proteins (MLH1, PMS2, MSH2, and MSH6).

\section{Methods}

Study approval was obtained from the Research Ethics Board at Mount Sinai Hospital.

Sixty consecutive cases of surgically resected colitisassociated primary colorectal adenocarcinoma from 58 patients between 2010 and 2018 were retrieved from the surgical pathology archives at Mount Sinai Hospital. Thirtytwo cases of surgically resected primary colorectal adenocarcinoma from patients without inflammatory bowel disease or any known hereditary cancer syndrome, hereforth termed sporadic colorectal carcinoma, were identified as a control group. H\&E sections were reviewed at a multiheaded microscope by four gastrointestinal pathologists (MI, HO, RR, and JC) and each invasive carcinoma was subclassified by consensus into one of five morphologic subtypes: conventional, mucinous, serrated, low grade tubuloglandular and others (Fig. 1).

At least one representative paraffin block of tumor was selected in each case for immunohistochemistry; if there 
Fig. 2 Immunohistochemical scoring of SATB2 expression. Examples of tumors scored as absent (a), weak (b), moderate (c), and strong (d) nuclear expression are shown
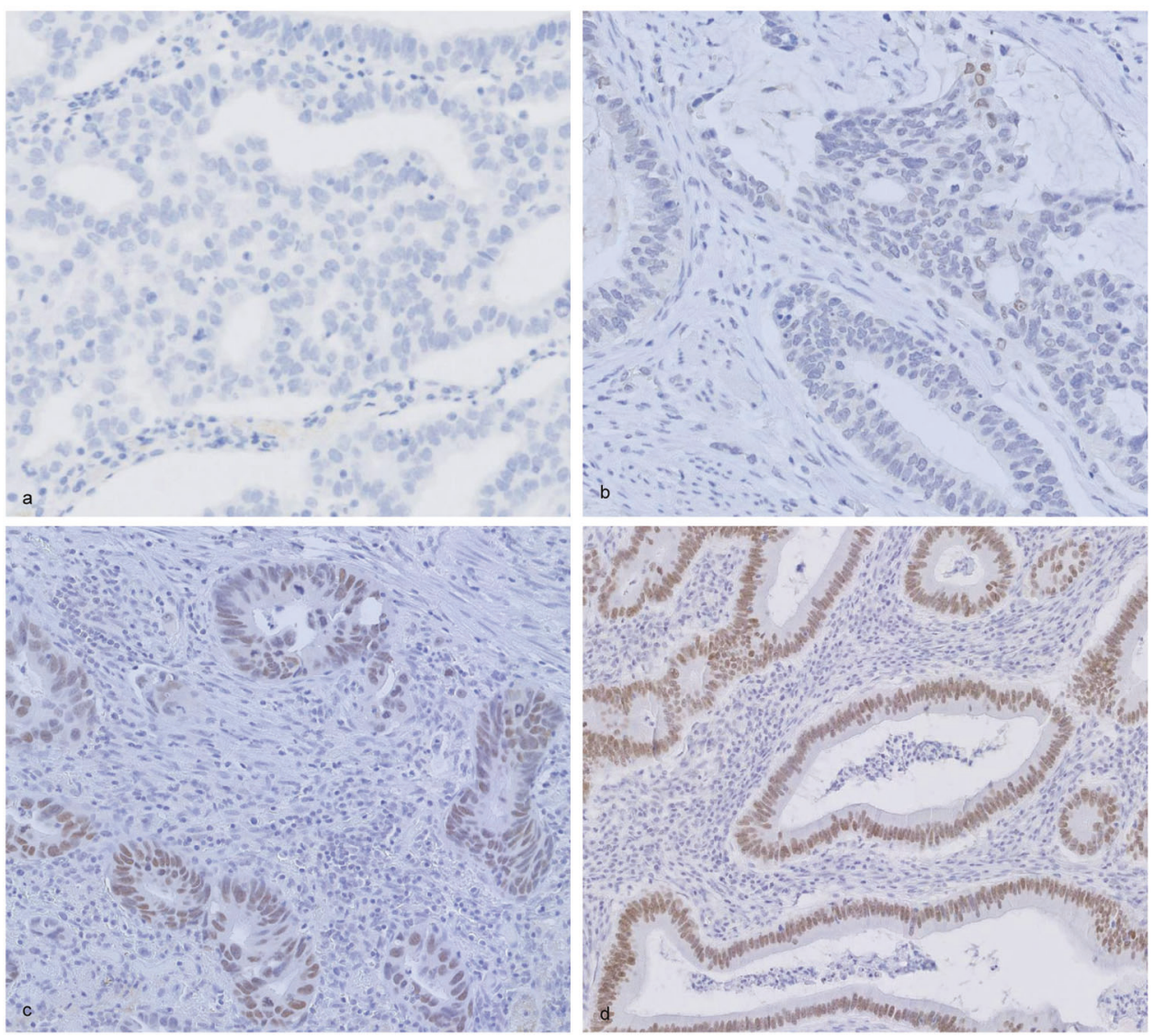

was significant morphologic heterogeneity in a given case, multiple tumor blocks were selected as needed to adequately represent the entire tumor. Immunohistochemical staining was performed using commercially available antibodies with the immuno-enzyme polymer method (Histofine Simple Stain MAX PO Multi, Nichirei Biosciences, Tokyo, Japan or Envision+System-HRP Labeled Polymer Anti-Rabbit, DAKO, Carpinteria, CA) with 3, 3'diaminobenzidone as the chromogen. The following primary antibodies were used in accordance with the manufacturers' instructions: SATB2 (clone: EPNCIR130A, abcam, Cambridge, UK), CDX2 (clone: CDX2-88, BioGenex, San Ramon, CA), MUC5AC antibody cocktail of two antibodies (CLH2, Novocastra, Newcastle-upon-Tyne, UK and 45M1, Novocastra), and MUC6 (clone: CLH5, Novocastra). Immunostaining was performed manually for all sporadic colorectal adenocarcinoma cases and 47 cases of CAC. Thirteen additional CAC cases were stained on an automated slide preparation system (SATB2, MUC5AC, MUC6: Dako Autostainer Plus; Agilent, Santa Clara, CA), (CDX2: Benchmark ULTRA; Ventana Medical Systems, Tucson, AZ).

Microsatellite-instability testing by immunohistochemistry for mismatch repair proteins (MLH1(clone: E505,
Novocastra), MSH2 (clone: FE11, Dako, Carpinteria, CA), MSH6(SP93, Cell Marque), and PMS2(clone MRQ-28, Cell Marque, Rocklin, CA) was conducted on an automated slide preparation system for $40 \mathrm{CAC}$ cases. In 20 cases, immunohistochemistry was manually performed (MLH1 (clone: E505, BD Biosciences, Franklin Lakes, NJ), MSH2 (clone: FE11, Merck KGaA, Darmstadt, Germany), MSH6 (EP49, Agilent), and PMS2 (EP51, Agilent).

The extent of staining for SATB2, CDX2, MUC5AC and MUC6 was scored semiquantitatively ( $0=$ no staining; $1 \leq$ $5 \% ; \quad 2=5-25 \% ; \quad 3=26-50 \% ; \quad 4=51-75 \% ; \quad 5=$ $76-100 \%$ ), and the maximum intensity was graded as negative [0], weak [1], moderate [2], or strong [3]. For binary analyses, cases with $5 \%$ or more tumor cells showing moderate or strong intensity were considered positive (Fig. 2). For MLH1, MSH2, MSH6, and PMS2, retained expression was defined as nuclear staining of any intensity within tumor cells, using infiltrating lymphocytes as a positive internal control. Deficient mismatch repair protein expression was defined as complete loss of expression of at least one of the 4 mismatch repair proteins.

Four of the authors (MI, HO, RR, and JC) reviewed the immunohistochemical stains at a multiheaded microscope and reached a consensus score for each case. 


\section{Statistics}

Chi-square test or Fisher exact tests were used to characterize the relationship between categorical variables. The Mann-Whitney $U$-test was used for comparisons between mean extent and intensity scores. Differences were considered to be significant at $p<0.05$. Statistical analyses were performed using StatFlex (Artech Co., Osaka, Japan).

\section{Results}

\section{Study group characteristics}

The 58 patients with colitis-associated carcinoma included 38 with ulcerative colitis and 20 with Crohn's disease. Two patients with ulcerative colitis had synchronous carcinomas for a total of 60 tumors evaluated. A summary of the clinicopathologic features of the colitis-associated carcinoma cohort and sporadic controls is presented in Table 1. The mean age at time of resection for patients with colitisassociated carcinoma was 50 years (range 24-76), significantly younger than sporadic cases (mean age 69 years; range $24-95 ; p<0.0001$ ). There was no significant difference in tumor location, $\mathrm{pT}$ stage, or $\mathrm{pN}$ stage between the colitis-associated carcinoma and sporadic carcinoma cohorts.

\section{Pathologic features and immunohistochemistry}

Colitis-associated carcinomas had a wider spectrum of tumor morphologies compared to sporadic colorectal adenocarcinomas. Only thirty-five of 60 (58\%) colitisassociated carcinomas had conventional morphology compared to $94 \%$ of sporadic cases $(p<0.001)$. Mucinous histology was significantly more frequent in colitisassociated carcinoma (20\% compared to $3 \%, p=0.029$ ). Although it was not statistically significant, 6 colitisassociated carcinomas (10\%) were subclassified into low grade tubuloglandular; none of the sporadic cases were of this subtype $(p=0.088)$. Of the two colitis-associated carcinoma cases subclassified as "other," one showed fetal enteric and hepatoid differentiation and another case showed mixed low grade tubuloglandular and serrated morphology in approximately equal proportions. Thirty-two percent of colitis-associated carcinomas were classified as overall high-grade compared to $6 \%$ of sporadic cases ( $p=$ 0.007; Table 1).

Only twenty-six of $60(43 \%)$ colitis associated carcinomas expressed SATB2 while, in contrast, expression was retained in 29 of $32(91 \%)$ sporadic colorectal adenocarcinomas $(p<0.0001$; Fig. 3$)$. The significant difference in SATB2 expression was retained in a subgroup analysis
Table 1 Clinicopathologic features of study group patients

\begin{tabular}{|c|c|c|c|}
\hline $\begin{array}{l}\text { Clinical and } \\
\text { pathologic } \\
\text { features }\end{array}$ & $\begin{array}{l}\text { Colitis-associated } \\
\text { colorectal } \\
\text { adenocarcinomas } \\
(N[\%])\end{array}$ & $\begin{array}{l}\text { Sporadic colorectal } \\
\text { adenocarcinomas } \\
(N[\%])\end{array}$ & $p$ \\
\hline No. of cases & 60 & 32 & \\
\hline $\begin{array}{l}\text { Sex, male/ } \\
\text { female }\end{array}$ & $33 / 27$ & $22 / 10$ & 0.201 \\
\hline $\begin{array}{l}\text { Mean age } \\
\text { (range) (years) }\end{array}$ & $50(24-76)$ & 69 (24-95) & $<0.0001$ \\
\hline \multicolumn{4}{|l|}{ Location } \\
\hline Right & $28(47)$ & $15(47)$ & 0.136 \\
\hline Left & $13(22)$ & $12(38)$ & \\
\hline Rectum & $19(32)$ & $5(16)$ & \\
\hline \multicolumn{4}{|l|}{ pT stage } \\
\hline 1 & $16(27)$ & $2(6)$ & 0.062 \\
\hline 2 & $8(13)$ & $9(28)$ & \\
\hline 3 & $25(42)$ & $16(50)$ & \\
\hline 4 & $11(18)$ & $5(16)$ & \\
\hline \multicolumn{4}{|l|}{$p N$ stage } \\
\hline 0 & $39(65)$ & $17(53)$ & 0.263 \\
\hline 1 or 2 & $21(35)$ & $15(47)$ & \\
\hline \multicolumn{4}{|l|}{ Cancer subtypes } \\
\hline Conventional & $35(58)$ & $30(94)$ & $<0.001$ \\
\hline Mucinous & $12(20)$ & $1(3)$ & 0.029 \\
\hline Serrated & $5(8)$ & $1(3)$ & 0.661 \\
\hline $\begin{array}{l}\text { Low grade } \\
\text { tubuloglandular }\end{array}$ & $6(10)$ & $0(0)$ & 0.088 \\
\hline Other & $2(3)$ & $0(0)$ & 0.541 \\
\hline \multicolumn{4}{|l|}{ Tumor grade } \\
\hline High & $19(32)$ & $2(6)$ & 0.007 \\
\hline Low & $41(68)$ & $30(94)$ & \\
\hline \multicolumn{4}{|c|}{ Immunohistochemistry } \\
\hline SATB 2 & $26(43)$ & $29(91)$ & $<0.0001$ \\
\hline CDX 2 & $52(87)$ & $32(100)$ & 0.047 \\
\hline MUC 5AC & $31(52)$ & $8(25)$ & 0.013 \\
\hline MUC 6 & $11(18)$ & $4(13)$ & 0.564 \\
\hline
\end{tabular}

comparing only colitis-associated carcinomas with conventional-type morphology to sporadic cases.

Among the 26 colitis-associated carcinomas that expressed SATB2, there was a significantly lower mean intensity score compared to positive sporadic cases (scores 2.58 and 2.86 , respectively; $p=0.018$ ). Mean extent of staining in the positive colitis-associated carcinomas was also lower, but this did not reach statistical significance (scores 4.42 and $4.83 ; p=0.097$ ). Within the colitisassociated carcinoma group, the number of mucinous and serrated cases that expressed SATB2 was too small for meaningful statistical analysis between multiple individual morphologic types. However, in a comparison between 

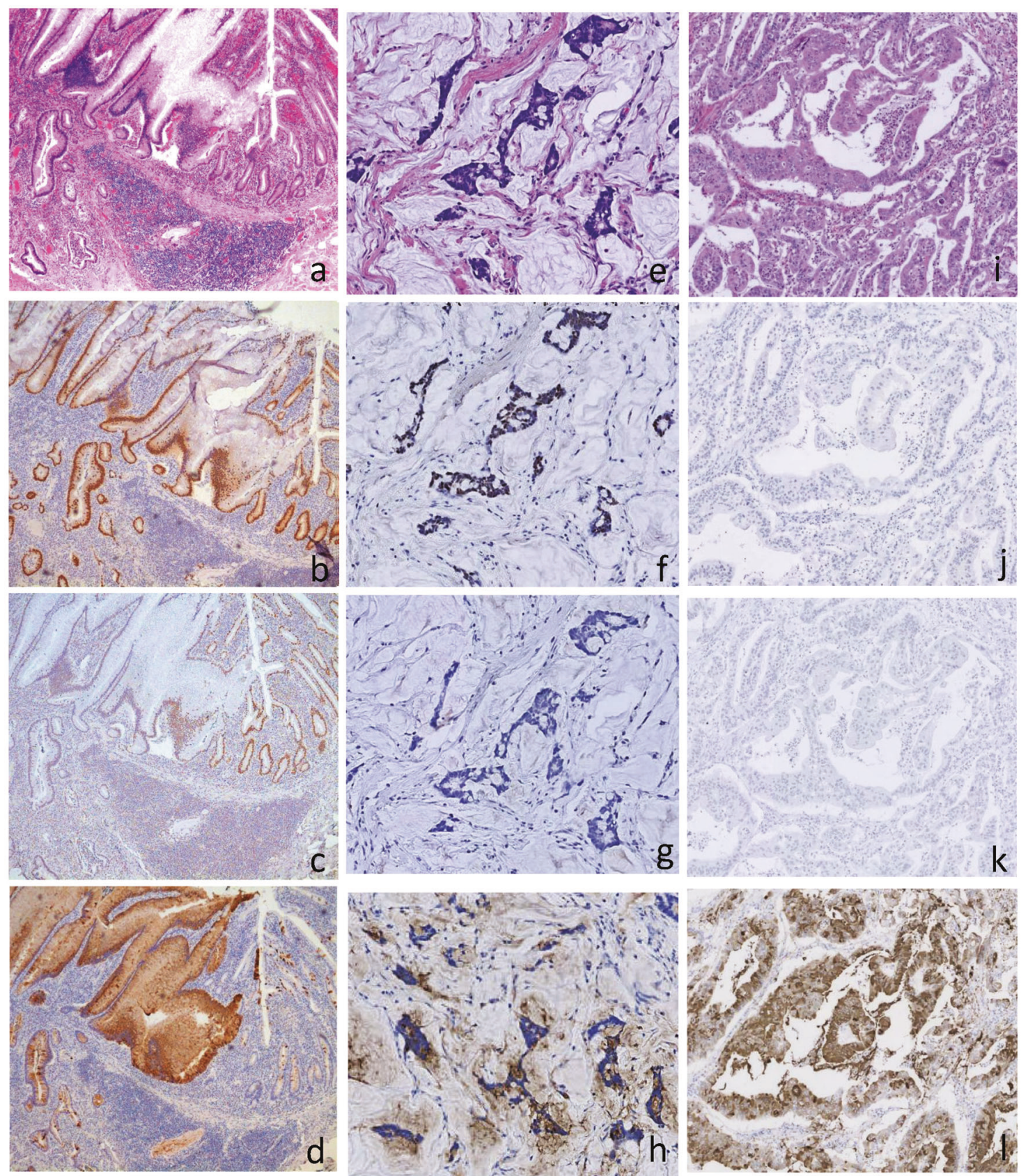

Fig. 3 Colitis-associated carcinoma with loss of SATB2 expression. Low grade tubuloglandular adenocarcinoma (a) with strong CDX2 expression (b), loss of SATB2 expression (retained in non-dysplastic colonic mucosa (c), and diffuse MUC5AC expression (d). Mucinous

conventional-type colitis associated carcinomas and all other morphologies, there was no significant difference in mean intensity score (2.61 and 2.50 , respectively; $p=$ 0.674 ) or mean extent score (4.28 and 4.78; $p=0.562)$.

The gastric foveolar-type mucin MUC5AC was expressed in thirty-one of 60 (52\%) colitis-associated carcinomas, which was significantly more frequent than in sporadic cases $(25 \%$; $p=0.013$; Fig. 3). 8 (13\%) cases of colitisassociated carcinoma showed loss of $\mathrm{CDX} 2$, whereas all adenocarcinoma (e), with CDX2 expression (f), loss of SATB2 expression (g), and MUC5AC expression (h). Serrated adenocarcinoma (i), with loss of CDX2 expression (j), loss of SATB2 expression (k) and diffuse MUC5AC expression (l)

sporadic colorectal adenocarcinomas retained nuclear expression $(p=0.047)$. No significant difference in MUC6 expression was identified. Seven cases of colitis-associated carcinoma were mismatch repair protein deficient and of these 2 were SATB2 negative. All 7 mismatch repair deficient cases had conventional-type morphology and retained CDX2 expression.

Although some degree of morphologic heterogeneity within lesions was common, two tumors had notably 
Table 2 Clinicopathologic features of ulcerative colitis and Crohn's disease-associated carcinomas

\begin{tabular}{|c|c|c|c|}
\hline $\begin{array}{l}\text { Clinical and } \\
\text { pathologic } \\
\text { features }\end{array}$ & $\begin{array}{l}\text { Ulcerative colitis- } \\
\text { associated } \\
\text { colorectal } \\
\text { adenocarcinoma } \\
(N[\%])\end{array}$ & $\begin{array}{l}\text { Crohn's disease- } \\
\text { associated } \\
\text { colorectal } \\
\text { adenocarcinoma } \\
(N[\%])\end{array}$ & $p$ \\
\hline No. of Cases & 40 & 20 & \\
\hline Sex, male/female & $25 / 15$ & $8 / 12$ & 0.099 \\
\hline $\begin{array}{l}\text { Mean age } \\
\text { (range) (years) }\end{array}$ & $50(24-70)$ & $50(24-76)$ & 0.544 \\
\hline \multicolumn{4}{|l|}{ Location } \\
\hline Right & $20(50)$ & $7(35)$ & 0.287 \\
\hline Left & $10(25)$ & $4(20)$ & \\
\hline Rectum & $10(25)$ & $9(45)$ & \\
\hline \multicolumn{4}{|l|}{$p T$ stage } \\
\hline 1 & $13(33)$ & $3(15)$ & 0.089 \\
\hline 2 & $6(15)$ & $2(10)$ & \\
\hline 3 & $16(40)$ & $7(35)$ & \\
\hline 4 & $5(13)$ & $8(40)$ & \\
\hline \multicolumn{4}{|l|}{$p N$ stage } \\
\hline 0 & $25(63)$ & $14(70)$ & 0.566 \\
\hline 1 or 2 & $15(38)$ & $6(30)$ & \\
\hline \multicolumn{4}{|l|}{ Cancer subtypes } \\
\hline Conventional & $27(68)$ & $8(40)$ & 0.042 \\
\hline Mucinous & $5(13)$ & $7(35)$ & 0.040 \\
\hline Serrated & $4(10)$ & $1(5)$ & 0.656 \\
\hline $\begin{array}{l}\text { Low grade } \\
\text { tubuloglandular }\end{array}$ & $3(8)$ & $3(15)$ & 0.653 \\
\hline Others & $1(3)$ & $1(5)$ & 1.00 \\
\hline \multicolumn{4}{|c|}{ Immunohistochemistry } \\
\hline SATB2 & $19(48)$ & $7(35)$ & 0.357 \\
\hline CDX 2 & $35(88)$ & $17(85)$ & 1.00 \\
\hline MUC 5AC & $21(53)$ & $10(50)$ & 0.855 \\
\hline MUC 6 & $8(20)$ & $2(10)$ & 0.471 \\
\hline $\begin{array}{l}\text { Mismatch repair } \\
\text { deficient }\end{array}$ & $4(10)$ & $3(15)$ & 0.676 \\
\hline
\end{tabular}

discrete transitions in morphology in different sections and stains were performed on multiple blocks. One of these lesions was a mucinous carcinoma that transitioned abruptly from low to high-grade and the other had a conventional component and a separate mucinous component. In both cases, the immunohistochemical profile in the different tumor areas was identical.

Crohn's disease-associated colorectal adenocarcinoma had a higher rate of mucinous histology compared to ulcerative colitis-associated carcinoma (35\% vs. $13 \%, p=$ 0.040 ) and ulcerative colitis-associated colorectal adenocarcinoma more frequently showed conventional morphology compared to Crohn's disease-associated colorectal adenocarcinoma ( $68 \%$ vs. $40 \%, p=0.041)$. There was no
Table 3 Clinicopathologic features of colitis-associated carcinomas with and without SATB2 expression

\begin{tabular}{|c|c|c|c|}
\hline $\begin{array}{l}\text { Clinical and } \\
\text { pathologic features }\end{array}$ & $\begin{array}{l}\text { SATB2 negative } \\
\text { colitis-associated } \\
\text { carcinoma }(N[\%])\end{array}$ & $\begin{array}{l}\text { SATB2 positive } \\
\text { colitis-associated } \\
\text { carcinoma }(N[\%])\end{array}$ & $p$ \\
\hline No. of cases & 34 & 26 & \\
\hline Sex, male/female & $20 / 14$ & $13 / 13$ & 0.496 \\
\hline $\begin{array}{l}\text { Mean age (range) } \\
\text { (years) }\end{array}$ & $50(24-76)$ & $50(24-70)$ & 0.875 \\
\hline \multicolumn{4}{|l|}{ Background } \\
\hline Ulcerative colitis & $21(62)$ & $19(73)$ & 0.357 \\
\hline Crohn's disease & $13(38)$ & $7(27)$ & \\
\hline \multicolumn{4}{|l|}{ Location } \\
\hline Right & $13(38)$ & $15(58)$ & 0.176 \\
\hline Left & $7(21)$ & $6(23)$ & \\
\hline Rectum & $14(41)$ & $5(19)$ & \\
\hline \multicolumn{4}{|l|}{$p T$ stage } \\
\hline 1 & $6(18)$ & $10(38)$ & 0.109 \\
\hline 2 & $3(9)$ & $5(19)$ & \\
\hline 3 & $17(50)$ & $8(31)$ & \\
\hline 4 & $8(24)$ & $3(12)$ & \\
\hline \multicolumn{4}{|l|}{$p N$ stage } \\
\hline 0 & $17(50)$ & $22(85)$ & 0.007 \\
\hline 1 or 2 & $17(50)$ & $4(15)$ & \\
\hline \multicolumn{4}{|l|}{ Cancer subtypes } \\
\hline Conventional & $17(50)$ & $18(69)$ & 0.134 \\
\hline Mucinous & $10(29)$ & $2(8)$ & 0.052 \\
\hline Serrated & $4(12)$ & $1(4)$ & 0.377 \\
\hline $\begin{array}{l}\text { Low grade } \\
\text { tubuloglandular }\end{array}$ & $2(6)$ & $4(15)$ & 0.388 \\
\hline Others & $1(3)$ & $1(4)$ & 1 \\
\hline \multicolumn{4}{|l|}{ Tumor grade } \\
\hline High & $13(38)$ & $6(23)$ & 0.211 \\
\hline Low & $21(62)$ & $20(77)$ & \\
\hline \multicolumn{4}{|c|}{ Immunohistochemistry } \\
\hline CDX 2 & $27(79)$ & $25(96)$ & 0.121 \\
\hline MUC 5AC & $20(59)$ & $11(42)$ & 0.205 \\
\hline MUC 6 & $7(21)$ & $4(15)$ & 0.742 \\
\hline $\begin{array}{l}\text { Mismatch repair } \\
\text { deficient }\end{array}$ & $2(6)$ & $5(19)$ & 0.222 \\
\hline
\end{tabular}

significant difference in the clinical features or immunoprofile between ulcerative colitis-associated and Crohn's disease-associated colorectal adenocarcinoma (Table 2).

\section{Association between SATB2 expression and clinicopathologic features in colitis associated carcinoma}

A summary of the clinicopathologic features of colitisassociated carcinoma cases is presented in Table 3.17 of 34 
(50\%) SATB2 negative colitis-associated carcinomas had lymph node metastasis compared to 4 of 26 (15\%) SATB2 positive cases $(p=0.007)$. SATB2 loss was particularly frequent in mucinous colitis-associated carcinoma, occurring in 10 of $12(83 \%)$ cases (Fig. 3). There was no significant association between SATB2 expression and histologic grade. Loss of CDX2 expression was strongly associated with concurrent SATB2 loss: of the 8 colitisassociated carcinomas that were negative for $\mathrm{CDX} 2,7$ $(88 \%)$ were also negative for SATB2. In contrast, most (79\%) SATB2 negative colitis-associated carcinomas retained CDX2 expression (Fig. 3).

Although colitis-associated carcinoma showed significantly more MUC5AC staining than sporadic colorectal adenocarcinoma, there was no significant difference in either MUC5AC or MUC6 expression between SATB2 negative colitis-associated carcinoma and SATB2 positive colitis-associated carcinoma (Table 3 ).

\section{Discussion}

SATB2 has been established as a diagnostically useful marker of colorectal (and appendiceal) origin in primary and metastatic carcinoma. While its sensitivity is comparable to CDX2, it is much more specific, with very limited staining in upper gastrointestinal tract, pancreaticobiliary, and ovarian intestinal-type carcinomas [8-10, 15].

Based on the accumulated evidence, at least $80 \%$ of colorectal adenocarcinomas are SATB2 positive. Magnusson et al reported that SATB2 is expressed in $85 \%$ of primary colorectal adenocarcinomas and $81 \%$ of metastatic colorectal adenocarcinomas (in addition to non-neoplastic colonic mucosa) [9]. Similarly, $\mathrm{Ma}$ et al. found positive SATB2 staining in $84 \%$ of colorectal adenocarcinomas. We found a similar rate of $91 \%$ in our sporadic colorectal adenocarcinoma control group. In contrast, however, there was a statistically and clinically significant lower rate of expression in colitis-associated carcinoma of only $43 \%$. These data have implications for workup of carcinoma of unknown primary origin in patients with a history of colitis. Absence of SATB2 immunoreactivity cannot reliably exclude colorectal origin in this group. One situation in which this consideration may be particularly relevant is in patients with primary sclerosing cholangitis-associated inflammatory bowel disease. In the presence of known cholangiocarcinoma, or, more commonly, radiographically indeterminate strictures, CDX2 expression without concurrent SATB2 staining should not be used to favor biliary over colorectal origin. What's more, $21 \%$ of SATB2 negative colitis-associated carcinomas in our study also lost expression of CDX2. Accordingly, SATB2 and CDX2 dual negative carcinomas should not entirely exclude colorectal origin in patients with inflammatory bowel disease.

There are some data suggesting that sporadic colorectal adenocarcinoma cases that exhibit loss of SATB2 expression are more likely to have lymph node metastasis and poorer overall prognosis [11, 12]. Eberhard et al reported that retained expression of SATB2 is an independent predictor of favorable outcome in colon cancer. Moreover, in curatively treated patients with stage III-IV disease, SATB2 expression is a predictor of response to adjuvant chemotherapy [11]. In our cohort, $50 \%$ of SATB2 negative colitis-associated carcinoma cases had lymph node metastasis compared to only $15 \%$ of cases with retained expression. Although interpretation of our results is limited by the unavailability of clinical follow-up data, given the frequency of SATB2 loss in colitis-associated carcinoma relative to sporadic colorectal adenocarcinoma, this could be a particularly useful prognostic marker for inflammatory bowel disease patients. Further study to include clinical outcomes is warranted to test this hypothesis.

This study also contributes an additional 58 cases to the published literature on clinicopathologic features of inflammatory bowel disease-associated carcinomas. Prior studies have shown that colitis associated carcinoma tends to affect younger patients [16], with average ages of 50-60 years in the setting of inflammatory bowel disease [3, 17], compared with colorectal adenocarcinoma in the general population, and has an increased frequency of mucinous histology [18]. Our cohort had similar findings, with a significantly younger mean patient age in colitis associated carcinoma and more frequent mucinous histology. Interestingly, some prior data have shown that mucinous histology is particularly frequent in Crohn's disease-associated colorectal adenocarcinoma [19] and in our study, a mucinous phenotype was significantly more common in Crohn's disease than ulcerative colitis, with the latter tending to have more conventional morphology. This is in contrast to one of the few studies directly comparing histological features of colorectal carcinoma in Crohn's disease and ulcerative colitis, which reported no significant histological difference between the two [20]. We found no differences between Crohn's disease and ulcerative colitis in $\mathrm{pT}$ stage, $\mathrm{pN}$ stage, or immunoprofile. Colitis-associated carcinoma cases with conventional morphology lost SATB2 expression at a rate similar to all colitis-associated carcinomas, and significantly more frequently than sporadic colorectal adenocarcinoma. This finding suggests that conventional morphology in colitis-associated carcinoma does not necessarily imply biologic overlap with sporadic colorectal adenocarcinoma.

Our data also support prior studies from smaller cohorts showing increased expression of gastric phenotype mucins in IBD associated adenocarcinoma [13, 14]. Such patterns have also been reported in non-neoplastic mucosa from 
inflammatory bowel disease patients, and have been suggested to arise from changes associated with regeneration of injured intestinal epithelium [21]. We found a significantly higher frequency of MUC5AC expression in colitisassociated carcinoma compared to sporadic colorectal adenocarcinoma, corroborating these data.

Our immunohistochemical data provide support at the protein expression level to recent studies demonstrating that most colitis-associated carcinoma cases do not arise through the conventional $A P C$ pathway $[4,22]$. Walsh et al. reported that mucin producing colorectal adenocarcinomas, with increased expression of MUC2, MUC5AC, and MUC6, along with loss of CDX2 expression, were associated with the serrated pathway of carcinogenesis. Loss of CDX2 expression is also strongly linked to CIMP-high, MLH1 methylation, and BRAF mutations, as well as MSI-high pathways [23]. Some studies have also described loss of CDX2 expression in MSI-high colorectal adenocarcinoma [24, 25]. In addition, Ma et al. [15] recently reported that loss of SATB2 expression in colorectal adenocarcinoma is associated with mismatch repair protein deficiency.

In spite of the higher frequency of mucinous and serrated morphology, significantly higher frequency of loss of SATB2 (and to a lesser degree CDX2) expression, and increased MUC5AC staining in our cohort of colitisassociated carcinoma, only 7 cases (12\%) were mismatch repair protein deficient, all of which had conventional morphology and retained CDX2 expression. SATB2 expression was not predictive of mismatch repair proteins status. Thus, while the precise molecular mechanism for the high frequency loss of SATB2 expression in colitisassociated carcinoma remains undefined, it appears to involve separate pathways from those previously reported to be associated with this finding in sporadic cases.

In conclusion, we demonstrate that, unlike sporadic colorectal carcinoma, loss of SATB2 expression is frequent in colitis-associated carcinoma, occurring in over $50 \%$ of cases. Additional features of colitis-associated carcinoma compared to sporadic controls include more frequent loss of CDX2 expression, mucinous histology, and MUC5AC immunoreactivity. Loss of SATB2 was particularly common in colitis-associated carcinoma with mucinous features, occurring in approximately $80 \%$ of these tumors, although there was no significant correlation with MUC5AC expression. In the setting of metastatic carcinoma from patients with inflammatory bowel disease, absence of SATB2 and CDX2 expression cannot exclude colorectal primary origin and should be interpreted cautiously. As in sporadic cases [11, 12], loss of SATB2 expression in colitisassociated carcinoma is associated with a higher rate of lymph node metastasis. While the specific molecular mechanisms underlying these findings are not understood, they demonstrate that the unique genetic underpinnings of colitis-associated carcinoma translate to differences at the histologic and immunohistochemical levels.

\section{Compliance with ethical standards}

Conflict of interest The authors declare that they have no conflict of interest.

Publisher's note: Springer Nature remains neutral with regard to jurisdictional claims in published maps and institutional affiliations.

\section{References}

1. Eaden JA, Abrams KR, Mayberry JF. The risk of colorectal cancer in ulcerative colitis: a meta-analysis. Gut. 2001;48:526-35.

2. Ekbom A, Helmick C, Zack M, Adami HO. Ulcerative colitis and colorectal cancer. A population-based study. $\mathrm{N}$ Engl J Med. 1990;323:1228-33.

3. Rutter MD, Saunders BP, Wilkinson KH, et al. Thirty-year analysis of a colonoscopic surveillance program for neoplasia in ulcerative colitis. Gastroenterology. 2006;130:1030-8.

4. Yaeger R, Shah MA, Miller VA, et al. Genomic alterations observed in colitis-associated cancers are distinct from those found in sporadic colorectal cancers and vary by type of inflammatory bowel disease. Gastroenterology. 2016;151:278-87.

5. Robles AI, Traverso G, Zhang M, et al. Whole-exome sequencing analyses of inflammatory bowel disease-associated colorectal cancers. Gastroenterology. 2016;150:931-43.

6. Dobreva G, Dambacher J, Grosschedl R. SUMO modification of a novel MAR-binding protein, SATB2, modulates immunoglobulin mu gene expression. Genes Dev. 2003;17:3048-61.

7. FitzPatrick DR, Carr IM, McLaren L, et al. Identification of SATB2 as the cleft palate gene on 2q32-q33. Hum Mol Genet. 2003;12:2491-501.

8. Lin F, Shi J, Zhu S, et al. Cadherin-17 and SATB2 are sensitive and specific immunomarkers for medullary carcinoma of the large intestine. Arch Pathol Lab Med. 2014;138:1015-26.

9. Magnusson K, de Wit M, Brennan DJ, et al. SATB2 in combination with cytokeratin 20 identifies over $95 \%$ of all colorectal carcinomas. Am J Surg Pathol. 2011;35:937-48.

10. Moh M, Krings G, Ates D, et al. SATB2 expression distinguishes ovarian metastases of colorectal and appendiceal origin from primary ovarian tumors of mucinous or endometrioid type. Am J Surg Pathol. 2016;40:419-32.

11. Eberhard J, Gaber A, Wangefjord S, et al. A cohort study of the prognostic and treatment predictive value of SATB2 expression in colorectal cancer. Br J Cancer. 2012;106:931-8.

12. Wang S, Zhou J, Wang XY, et al. Down-regulated expression of SATB2 is associated with metastasis and poor prognosis in colorectal cancer. J Pathol. 2009;219:114-22.

13. Tatsumi N, Kushima R, Vieth M, et al. Cytokeratin $7 / 20$ and mucin core protein expression in ulcerative colitis-associated colorectal neoplasms. Virchows Arch. 2006;448:756-62.

14. Whitcomb E, Liu X, Xiao SY. Crohn enteritis-associated small bowel adenocarcinomas exhibit gastric differentiation. Hum Pathol. 2014;45:359-67.

15. Ma C, Olevian DC, Lowenthal BM, et al. Loss of SATB2 expression in colorectal carcinoma is associated with DNA mismatch repair protein deficiency and BRAF mutation. Am J Surg Pathol. 2018;42:1409-17.

16. Jess T, Rungoe C, Peyrin-Biroulet L. Risk of colorectal cancer in patients with ulcerative colitis: a meta-analysis of 
population-based cohort studies. Clin Gastroenterol Hepatol. 2012;10:639-45.

17. Baars JE, Kuipers EJ, van Haastert M, et al. Age at diagnosis of inflammatory bowel disease influences early development of colorectal cancer in inflammatory bowel disease patients: a nationwide, long-term survey. J Gastroenterol. 2012;47:1308-22.

18. Itzkowitz SH, Yio X. Inflammation and cancer IV. Colorectal cancer in inflammatory bowel disease: the role of inflammation. Am J Physiol Gastrointest Liver Physiol. 2004;287:G7-17.

19. Galata C, Hirsch D, Reindl W, et al. Clinical and histopathologic features of colorectal adenocarcinoma in Crohn's disease. J Clin Gastroenterol. 2018;52:635-40.

20. Svrcek M, Cosnes J, Beaugerie L, et al. Colorectal neoplasia in Crohn's colitis: a retrospective comparative study with ulcerative colitis. Histopathology. 2007;50:574-83.

21. Shaoul R, Okada Y, Cutz E, Marcon MA. Colonic expression of MUC2, MUC5AC, and TFF1 in inflammatory bowel disease in children. J Pediatr Gastroenterol Nutr. 2004;38:488-93.
22. Hartman DJ, Binion DG, Regueiro MD, et al. Distinct histopathologic and molecular alterations in inflammatory bowel disease-associated intestinal adenocarcinoma: c-MYC amplification is common and associated with mucinous/signet ring cell differentiation. Inflamm Bowel Dis. 2018;24:1780-90.

23. Walsh MD, Clendenning M, Williamson E, et al. Expression of MUC2, MUC5AC, MUC5B, and MUC6 mucins in colorectal cancers and their association with the $\mathrm{CpG}$ island methylator phenotype. Mod Pathol. 2013;26:1642-56.

24. Kim JH, Rhee YY, Bae JM, Cho NY, Kang GH. Loss of CDX2/ CK20 expression is associated with poorly differentiated carcinoma, the $\mathrm{CpG}$ island methylator phenotype, and adverse prognosis in microsatellite-unstable colorectal cancer. Am J Surg Pathol. 2013;37:1532-41.

25. Lugli A, Tzankov A, Zlobec I, Terracciano LM. Differential diagnostic and functional role of the multi-marker phenotype CDX2/CK20/CK7 in colorectal cancer stratified by mismatch repair status. Mod Pathol. 2008;21:1403-12. 